\title{
A Simple Proof of Hardy-Lieb-Thirring Inequalities
}

\section{Rupert L. Frank}

Department of Mathematics, Princeton University, Princeton,

NJ 08544, USA. E-mail: rlfrank@math.princeton.edu

Received: 19 September 2008 / Accepted: 18 November 2008

Published online: 13 March 2009 - (C) The Author 2009

Abstract: We give a short and unified proof of Hardy-Lieb-Thirring inequalities for moments of eigenvalues of fractional Schrödinger operators. The proof covers the optimal parameter range. It is based on a recent inequality by Solovej, Sørensen, and Spitzer. Moreover, we prove that any non-magnetic Lieb-Thirring inequality implies a magnetic Lieb-Thirring inequality (with possibly a larger constant).

\section{Introduction and main result}

This paper is concerned with estimates on moments of negative eigenvalues of Schrödinger operators $(-\Delta)^{s}-\mathcal{C}_{s, d}|x|^{-2 s}-V$ in $L_{2}\left(\mathbb{R}^{d}\right)$ in terms of integrals of the potential $V$. Here

$$
\mathcal{C}_{s, d}:=2^{2 s} \frac{\Gamma((d+2 s) / 4)^{2}}{\Gamma((d-2 s) / 4)^{2}}
$$

is the sharp constant in the Hardy inequality

$$
\int_{\mathbb{R}^{d}}|p|^{2 s}|\hat{u}(p)|^{2} d p \geq \mathcal{C}_{s, d} \int_{\mathbb{R}^{d}}|x|^{-2 s}|u(x)|^{2} d x, \quad u \in C_{0}^{\infty}\left(\mathbb{R}^{d}\right),
$$

which is valid for $0<s<d / 2[\mathrm{He}]$ and we write $\hat{u}(p):=(2 \pi)^{-d / 2} \int_{\mathbb{R}^{d}} u(p) e^{-i p \cdot x} d x$ for the Fourier transform of $u$. In [FrLiSe1] we have shown that for any $\gamma>0,0<s \leq 1$ and $0<s<d / 2$ one has

$$
\operatorname{tr}\left((-\Delta)^{s}-\mathcal{C}_{s, d}|x|^{-2 s}-V\right)_{-}^{\gamma} \leq L_{\gamma, d, s}^{\mathrm{HLT}} \int_{\mathbb{R}^{d}} V(x)_{+}^{\gamma+d / 2 s} d x
$$


with a constant $L_{\gamma, d, s}^{\mathrm{HLT}}$ independent of $V$. Here and in the following, $t_{ \pm}:=\max \{ \pm t, 0\}$ denote the positve and negative parts of a real number or a self-adjoint operator $t$. The case $s=1$ in (1.3) has been shown earlier in [EkFr]. We refer to (1.3) as HardyLieb-Thirring inequality since it is (up to the value of the constant) an improvement of the Lieb-Thirring inequality [LiTh]

$$
\operatorname{tr}\left((-\Delta)^{s}-V\right)_{-}^{\gamma} \leq L_{\gamma, d, s} \int_{\mathbb{R}^{d}} V(x)_{+}^{\gamma+d / 2 s} d x .
$$

It should be pointed out that if $0<s<d / 2$, then (1.4) is valid even for $\gamma=0$ (as first shown by Cwikel, Lieb, and Rozenblum) while (1.3) is not. We refer to the surveys $[\mathrm{LaWe}, \mathrm{Hu}]$ for background and references concerning (1.4).

The original motivation for (1.4) came from the problem of stability of nonrelativistic matter (see [LiSe] for a textbook presentation). Likewise, our motivation for (1.3) was stability of relativistic matter in magnetic fields. For this problem it is crucial that (1.3) continues to hold if $(-\Delta)^{s}$ is replaced by $|D-A|^{2 s}$ with a magnetic vector potential $A \in L_{2, \text { loc }}\left(\mathbb{R}^{d}, \mathbb{R}^{d}\right)$, and that the constant can be chosen independently of $A$. Here, as usual, $D=-i \nabla$ and the operator $|D-A|^{2 s}:=\left((D-A)^{2}\right)^{s}$ is defined by means of the spectral theorem. Using the magnetic version of (1.3) we could prove stability of relativistic matter in magnetic fields up to and including the critical value of the nuclear charge $\alpha Z=2 / \pi=\mathcal{C}_{1 / 2,3}$; see [FrLiSe1] and also [FrLiSe2].

The purpose of this paper is fourfold.

(1) We will give a new, much simpler proof of (1.3). While the method in [FrLiSe1] relied on rather involved relations between Sobolev inequalities and decay estimates on heat kernels, the present proof uses nothing more than (1.4) (with $\gamma=0$ and with $s$ replaced by some $t<s$ ) and the generalization of a powerful (though elementary to prove) new inequality by Solovej, Sørensen and Spitzer [SoSøSp].

(2) We will extend (1.3) to its optimal parameter range $0<s<d / 2$. For $d \geq 3$ and $1<s<d / 2$ this is a new result, even for integer values of $s$ when the operator is local. This result can not be attained with the method of [FrLiSe1], since positivity properties of the heat kernel break down for $s>1$.

(3) Though our new proof of (1.3) does not work in the presence of a magnetic field, we shall prove a new operator-theoretic result, which says that any non-magnetic Lieb-Thirring inequality implies a magnetic Lieb-Thirring inequality (with possibly a different constant). This recovers, in particular, that (1.3) holds if $(-\Delta)^{s}$ is replaced by $|D-A|^{2 s}$ and $0<s \leq 1$. (The reason for the restriction $s \leq 1$ at this point is that we need a diamagnetic inequality.) Another application of this result concerns the recent inequality in [KoVuWe] corresponding to the endpoint $\gamma=0$ of (1.4) with $s=1, d=2$.

(4) We show that an analog of inequality (1.3) for $s=1 / 2, d=3$ holds in a model for pseudo-relativistic electrons that includes spin. The difficulty here is that the potential energy is non-local. This new estimate simplifies some of the arguments in [FrSiWa] and will be, we believe, a crucial ingredient in the proof of stability of matter in this model.

Here is the precise statement of our result.

Theorem 1.1. Let $d \geq 1,0<s<d / 2$ and $\gamma>0$. Then there is a constant $L_{\gamma, d, s}^{\mathrm{HLT}}$ such that

$$
\operatorname{tr}\left((-\Delta)^{s}-\mathcal{C}_{s, d}|x|^{-2 s}-V\right)_{-}^{\gamma} \leq L_{\gamma, d, s}^{\mathrm{HLT}} \int_{\mathbb{R}^{d}} V(x)_{+}^{\gamma+d / 2 s} d x
$$


If $d \geq 2,0<s \leq 1$ and $(-\Delta)^{s}$ is replaced by $|D-A|^{2 s}$ for some $A \in L_{2, \mathrm{loc}}\left(\mathbb{R}^{d}, \mathbb{R}^{d}\right)$, then (1.5) remains valid if $L_{\gamma, d, s}^{\mathrm{HLT}}$ is replaced by $L_{\gamma, d, s}^{\mathrm{HLT}}(e / p)^{p} \Gamma(p+1)$ with $p=\gamma+d / 2 s$.

The crucial ingredient in our proof of (1.5) is the following lower bound for the quadratic form

$$
h_{s}[u]:=\int_{\mathbb{R}^{d}}|p|^{2 s}|\hat{u}(p)|^{2} d p-\mathcal{C}_{s, d} \int_{\mathbb{R}^{d}}|x|^{-2 s}|u(x)|^{2} d x
$$

of the operator $(-\Delta)^{s}-\mathcal{C}_{s, d}|x|^{-2 s}$.

Theorem 1.2. Let $0<t<s<d / 2$. Then there exists a constant $\kappa_{d, s, t}>0$ such that for all $u \in C_{0}^{\infty}\left(\mathbb{R}^{d}\right)$ one has

$$
h_{s}[u]^{\theta}\|u\|^{2(1-\theta)} \geq \kappa_{d, s, t}\left\|(-\Delta)^{t / 2} u\right\|^{2}, \quad \theta:=t / s .
$$

In the special case $d=3$ and $s=1 / 2$ this is a recent result by Solovej, Sørensen and Spitzer [SoSøSp, Thm. 11]. The results reported here are motivated by their work. Below we shall show that their proof extends to arbitrary $0<s<d / 2$.

Our original proof of (1.5) in [FrLiSe1] for $0<s \leq 1$ relied on the GagliardoNirenberg-type inequality

$$
h_{s}[u]^{\theta}\|u\|^{2(1-\theta)} \geq \sigma_{d, s, q}\|u\|_{q}^{2}, \quad \theta:=\frac{d}{s}\left(\frac{1}{2}-\frac{1}{q}\right),
$$

for $2<q<2 s /(d-2 s)$. This is weaker than (1.6) in view of the Sobolev inequality [LiLo, Thms. 4.3 and 8.3]

$$
\left\|(-\Delta)^{t / 2} u\right\|^{2} \geq S_{d, t}\|u\|_{q}^{2}, \quad q=\frac{2 d}{d-2 t} .
$$

What makes (1.6) much easier to prove than (1.7) is that it is a linear inequality, that is, all norms are taken in $L_{2}\left(\mathbb{R}^{d}\right)$. Indeed, (1.6) is easily seen to be equivalent to the operator inequality

$$
(-\Delta)^{s}-\mathcal{C}_{s, d}|x|^{-2 s} \geq K_{d, s, t} l^{-2(s-t)}(-\Delta)^{t}-l^{-2 s}, \quad l>0,
$$

where $K_{d, s, t}=\left(s^{-s} t^{t}(s-t)^{s-t}\right)^{1 / s} \kappa_{d, s, t}$, and this is the way we shall prove it in the next section.

\section{Proof of Theorem 1.2}

Throughout this section we assume that $0<s<d / 2$. Recall that for $0<\alpha<d$ the Fourier transform of $|x|^{-d+\alpha}$ is given by

$$
b_{d-\alpha}\left(|\cdot|^{-d+\alpha}\right)^{\wedge}(p)=b_{\alpha}|p|^{-\alpha}, \quad b_{\alpha}:=2^{\alpha / 2} \Gamma(\alpha / 2) ;
$$

see, e.g., [LiLo, Thm. 5.9], where another convention for the Fourier transform is used, however. This implies that for $2 s<\alpha<d$ one has

$$
\int_{\mathbb{R}^{d}} \frac{1}{|p-q|^{d-2 s}|q|^{\alpha}} d q=\Psi_{s, d}(\alpha) \frac{1}{|p|^{\alpha-2 s}},
$$


where

$\Psi_{s, d}(\alpha):=(2 \pi)^{d / 2} \frac{b_{2 s} b_{\alpha-2 s} b_{d-\alpha}}{b_{d-2 s} b_{d-\alpha+2 s} b_{\alpha}}=\frac{\pi^{d / 2} \Gamma(s)}{\Gamma((d-2 s) / 2)} \frac{\Gamma((\alpha-2 s) / 2) \Gamma((d-\alpha) / 2)}{\Gamma((d-\alpha+2 s) / 2) \Gamma(\alpha / 2)}$.

We shall need the following facts about $\Psi_{s, d}(\alpha)$ as a function of $\alpha \in(2 s, d)$.

Lemma 2.1. $\Psi_{s, d}$ is an even function with respect to $\alpha=(d+2 s) / 2$ and one has

$$
\Psi_{s, d}((d+2 s) / 2)=(2 \pi)^{d / 2} \frac{b_{2 s}}{b_{d-2 s}} \mathcal{C}_{s, d}^{-1}
$$

with $\mathcal{C}_{s, d}$ from (1.1). Moreover, $\Psi_{s, d}$ is strictly decreasing on $(2 s,(d+2 s) / 2)$ and strictly increasing on $((d+2 s) / 2, d)$.

This is Lemma 3.2 from [FrLiSe1] in disguise.

Proof of Lemma 2.1. $\Psi_{s, d}(\alpha)$ is obviously invariant under replacing $\alpha$ by $d+2 s-\alpha$, and its value at $\alpha=(d+2 s) / 2$ follows immediately from definition (1.1). To prove the monotonicity we write

$$
\Psi_{s, d}(\alpha)=\frac{\pi^{d / 2} \Gamma(s)}{\Gamma((d-2 s) / 2)} \frac{f(t)}{f(s+t)}, \quad t=(\alpha-2 s) / 2,
$$

where $T:=(d-2 s) / 2$ and $f(t):=\Gamma(t) / \Gamma(T+s-t)$. We need to show that $\log (f(t) / f(s+t))$ is strictly decreasing in $t \in(0, T / 2)$. Noting that

$$
\frac{f^{\prime}(t)}{f(t)}=\psi(t)+\psi(T+s-t)
$$

with $\psi:=\Gamma^{\prime} / \Gamma$ the Digamma function, we have

$$
\frac{d}{d t} \log \frac{f(t)}{f(t+s)}=\psi(t)+\psi(T+s-t)-\psi(t+s)-\psi(T-t)=-\int_{t}^{t+s} h(\tau) d \tau
$$

with $h(\tau):=\psi^{\prime}(\tau)-\psi^{\prime}(T+s-\tau)$ for $0<\tau<T+s$. Since $\psi^{\prime}$ is strictly decreasing [AbSt, (6.4.1)], $h$ is an odd function with respect to $\tau=(T+s) / 2$ which is strictly positive for $\tau<(T+s) / 2$. Since the midpoint of the interval $(t, t+s)$ lies to the left of $(T+s) / 2$, the integral of $h$ over this interval is strictly positive, which proves the claim.

Now we prove (1.8), following the strategy of Solovej, Sørensen and Spitzer [SoSøSp] in the special case $d=3, s=1 / 2$; see also [LiYa, Thm. 11] for a related argument.

Proof of Theorem 1.2. For technical reasons we prove the theorem only for $2 s / 3 \leq t<s$. In view of the inequality $(-\Delta)^{t} \leq(3 t / 2 s) l^{2(s-3 t / 2)}(-\Delta)^{2 s / 3 t}+(1-3 t / 2 s) l^{-2 t}$ for $t<2 s / 3$ and $l>0$ this implies the result for all $0<t<s$.

By a well-known argument (going back at least to Abel and, in the present context, to [KoPeSe]) based on the Cauchy-Schwarz inequality, one has for any positive measurable function $h$ on $\mathbb{R}^{d}$

$$
(2 \pi)^{d / 2} \frac{b_{2 s}}{b_{d-2 s}} \int_{\mathbb{R}^{d}} \frac{|u|^{2}}{|x|^{2 s}} d x=\iint_{\mathbb{R}^{d} \times \mathbb{R}^{d}} \frac{\overline{\hat{u}(p)} \hat{u}(q)}{|p-q|^{d-2 s}} d p d q \leq \int_{\mathbb{R}^{d}} t_{h}(p)|\hat{u}(p)|^{2} d p,
$$


where

$$
t_{h}(p):=h(p)^{-1} \int_{\mathbb{R}^{d}} \frac{h(q)}{|p-q|^{d-2 s}} d q .
$$

Below we shall choose $h$ (depending on $l>0$ ) in such a way that for some positive constants $A$ and $B$ (depending on $d, s$ and $t$, but not on $l$ ) one has

$$
t_{h}(p) \leq \Psi_{s, d}((d+2 s) / 2)|p|^{2 s}-A l^{-2(s-t)}|p|^{2 t}+B l^{-2 s} .
$$

(By scaling it would be enough to prove this for $l=1$, but we prefer to keep $l$ free.) Because of (2.3) this estimate proves (1.8).

We show that (2.4) holds with $h(p)=\left(|p|^{(d+2 s) / 2}+l^{\beta-(d+2 s) / 2}|p|^{\beta}\right)^{-1}$, where $\beta$ is a parameter depending on $t$ that will be fixed later. (Indeed, we shall choose $\beta=2 t+(d-2 s) / 2$.) Since the derivatives of the function $r \mapsto r^{-1}$ have alternating signs one has $(a+b)^{-1} \leq a^{-1}-a^{-2} b+a^{-3} b^{2}$ and therefore

$$
\begin{aligned}
& \int_{\mathbb{R}^{d}} \frac{h(q)}{|p-q|^{d-2 s}} d q \\
& \quad \leq \int_{\mathbb{R}^{d}} \frac{1}{|p-q|^{d-2 s}}\left(\frac{1}{|q|^{(d+2 s) / 2}}-\frac{l^{\beta-(d+2 s) / 2}}{|q|^{d+2 s-\beta}}+\frac{l^{2 \beta-d-2 s}}{|q|^{3(d+2 s) / 2-2 \beta}}\right) d q .
\end{aligned}
$$

If we assume that $(d+6 s) / 4<\beta<(3 d+2 s) / 4$ then the right side is finite and, using notation (2.2) with $\Psi$ instead of $\Psi_{s, d}$, equal to

$$
\begin{aligned}
& \Psi\left(\frac{d+2 s}{2}\right) \frac{1}{|p|^{(d-2 s) / 2}}-\Psi(d+2 s-\beta) \frac{l^{\beta-(d+2 s) / 2}}{|p|^{d-\beta}} \\
& +\Psi\left(\frac{3(d+2 s)}{2}-2 \beta\right) \frac{l^{2 \beta-d-2 s}}{|p|^{3 d / 2-2 \beta+s}} .
\end{aligned}
$$

Thus

$$
\begin{aligned}
t_{h}(p) \leq \Psi & \left(\frac{d+2 s}{2}\right)|p|^{2 s}-\left(\Psi(d+2 s-\beta)-\Psi\left(\frac{d+2 s}{2}\right)\right) l^{\beta-(d+2 s) / 2}|p|^{\beta-(d-2 s) / 2} \\
& +\left(\Psi\left(\frac{3(d+2 s)}{2}-2 \beta\right)-\Psi(d+2 s-\beta)\right) l^{2 \beta-d-2 s}|p|^{2 \beta-d} \\
& +\Psi\left(\frac{3(d+2 s)}{2}-2 \beta\right) l^{3 \beta-3 d / 2-3 s}|p|^{3 \beta-3 d / 2-s} .
\end{aligned}
$$

If we assume that $\beta \leq(d+2 s) / 2$, then the exponents of $|p|$ on the right side satisfy $2 s \geq \beta-(d-2 s) / 2 \geq 2 \beta-d \geq 3 \beta-3 d / 2-s$, and if $\beta \geq(3 d+2 s) / 6$ then the last exponent is non-negative. Now we choose $\beta=2 t+(d-2 s) / 2$, so that the exponent of the second term is $2 t$ and the condition $\beta \geq(3 d+2 s) / 6$ is satisfied, since we are assuming that $t \geq 2 s / 3$. Moreover, according to Lemma 2.1, the coefficient of the second term is negative. Finally, we use that there are constants $C_{1}$ and $C_{2}$ such that for any $\varepsilon>0$ one has

$$
\begin{gathered}
|p|^{2 \beta-d} \leq \varepsilon|p|^{\beta-(d-2 s) / 2}+C_{1} \varepsilon^{-\frac{2(2 \beta+d)}{d+2 s-2 \beta}}, \\
|p|^{3 \beta-3 d / 2-s} \leq \varepsilon|p|^{\beta-(d-2 s) / 2}+C_{2} \varepsilon^{-\frac{6 \beta-3 d-2 s}{2(d+2 s-2 \beta)}} .
\end{gathered}
$$

This concludes the proof of (2.4). 


\section{Proof of Theorem 1.1}

We fix $0<s<d / 2$ and $\gamma>0$ and write

$\operatorname{tr}\left((-\Delta)^{s}-\mathcal{C}_{s, d}|x|^{-2 s}-V\right)_{-}^{\gamma}=\gamma \int_{0}^{\infty} N\left(-\tau,(-\Delta)^{s}-\mathcal{C}_{s, d}|x|^{-2 s}-V\right) \tau^{\gamma-1} d \tau$,

where $N(-\tau, H)$ denotes the number of eigenvalues less than $-\tau$, counting multiplicities, of a self-adjoint operator $H$. We shall use (1.8) with $l^{-2 s}=\sigma \tau$ and some $0<t<s$ and $0<\sigma<1$ to be specified below. Abbreviating $K_{t}=K_{d, s, t}$ we find that

$$
\begin{aligned}
N\left(-\tau,(-\Delta)^{s}-\mathcal{C}_{s, d}|x|^{-2 s}-V\right) & \leq N\left(0, K_{t}(\sigma \tau)^{(s-t) / s}(-\Delta)^{t}-V+(1-\sigma) \tau\right) \\
& =N\left(0,(-\Delta)^{t}-K_{t}^{-1}(\sigma \tau)^{-(s-t) / s}(V-(1-\sigma) \tau)\right) .
\end{aligned}
$$

Now we use (1.4) with $\gamma=0$ and $s$ replaced by $t$ (see [Da] for $t \leq 1$ and [Cw] for $t<d / 2$ ). Abbreviating $L_{t}=L_{0, d, t}$ we have

$$
\begin{aligned}
& N\left(-\tau,(-\Delta)^{s}-\mathcal{C}_{s, d}|x|^{-2 s}-V\right) \\
& \quad \leq L_{t} K_{t}^{-d / 2 t}(\sigma \tau)^{-d(s-t) / 2 s t} \int_{\mathbb{R}^{d}}(V-(1-\sigma) \tau)_{+}^{d / 2 t} d x
\end{aligned}
$$

and

$$
\begin{aligned}
& \operatorname{tr}\left((-\Delta)^{s}-\mathcal{C}_{s, d}|x|^{-2 s}-V\right)_{-}^{\gamma} \\
& \quad \leq \gamma L_{t} K_{t}^{-d / 2 t} \sigma^{-d(s-t) / 2 s t} \int_{\mathbb{R}^{d}} d x \int_{0}^{\infty} d \tau \tau^{\gamma-1-d(s-t) / 2 s t}(V-(1-\sigma) \tau)_{+}^{d / 2 t} d x \\
& \quad=\gamma L_{t} K_{t}^{-d / 2 t} \sigma^{-\frac{d(s-t)}{2 s t}}(1-\sigma)^{-\gamma+\frac{d(s-t)}{2 s t}} \frac{\Gamma\left(\gamma-\frac{d(s-t)}{2 s t}\right) \Gamma\left(\frac{d}{2 t}+1\right)}{\Gamma\left(\gamma+\frac{d}{2 s}+1\right)} \int_{\mathbb{R}^{d}} V_{+}^{\gamma+d / 2 s} d x .
\end{aligned}
$$

Here we assumed that $t>d s /(2 \gamma s+d)$ so that the $\tau$ integral is finite. Finally, we optimize over $0<\sigma<1$ by choosing $\sigma=d(s-t) / 2 \gamma s t$ and over $d s /(2 \gamma s+d)<t<s$ to complete the proof of (1.5).

The statement about the inclusion of $A$ follows from Example 4.1 and Theorem 4.2 in the following section.

\section{Magnetic Lieb-Thirring Inequalities}

In this section we discuss Lieb-Thirring inequalities for magnetic Schrödinger operators, that is, (1.4) (and its generalizations) with $(-\Delta)^{s}$ replaced by $|D-A|^{2 s}$ for some vector field $A \in L_{2, \text { loc }}\left(\mathbb{R}^{d}, \mathbb{R}^{d}\right)$.

It is a remarkable fact that all presently known proofs of Lieb-Thirring inequalities, which allow for the inclusion of a magnetic field, yield the same constants in the magnetic case as in the non-magnetic case. It is unknown whether this is also true for the unknown sharp constants. Note that the diamagnetic inequality implies that the lowest eigenvalue does not decrease when a magnetic field is added, but there is no such result for, e.g., the number or the sum of eigenvalues; see [AvHeSi,Li]. Rozenblum [Ro] discovered, however, that any power-like bound on the number of eigenvalues in the non-magnetic case implies a similar bound in the magnetic case, with possibly a worse constant. Here we show the same phenomenon for moments of eigenvalues. 
We work in the following abstract setting. Let $(X, \mu)$ be a sigma-finite measure space and let $H$ and $M$ be non-negative operators in $L_{2}(X, \mu)$ such that for any $u \in L_{2}(X, \mu)$ and any $t>0$,

$$
|\exp (-t M) u(x)| \leq(\exp (-t H)|u|)(x) \quad \mu-\text { a.e. } x \in X .
$$

Note that this implies that $\exp (-t H)$ is positivity preserving. We think of $H$ as a non-magnetic operator, $M$ a magnetic operator and (4.1) as a diamagnetic inequality. It might be useful to keep the following example in mind.

Example 4.1. Let $X=\mathbb{R}^{d}$ with Lebesgue measure, $H=(-\Delta)^{s}$, and $M=|D-A|^{2 s}$ for some $0<s \leq 1$ and $A \in L_{2, \text { loc }}\left(\mathbb{R}^{d}\right)$. The diamagnetic inequality (4.1) in the case $s=1$ was shown in [Si1], and in the case $0<s<1$ it follows from the $s=1$ result since the function $\lambda \mapsto \exp \left(-\lambda^{S}\right)$ is completely monotone and hence by Bernstein's theorem [Do] the Laplace transform of a positive measure. More generally, (4.1) holds for $H=(-\Delta)^{s}+W$ and $M=|D-A|^{2 s}+W$ with $s$ and $A$ as before and a, say, bounded function $W$. This can be seen using Trotter's product formula. By an approximation argument the inequality holds also for $W(x)=-\mathcal{C}_{s, d}|x|^{-2 s}$; see [FrLiSe1].

The main result in this section is

Theorem 4.2. Let $H$ and $M$ be as above and assume that there exist some constants $L>$ $0, \gamma \geq 0, p>0$ and a non-negative function $w$ on $X$ such that for all $V \in L_{p}(V, w d \mu)$ one has

$$
\operatorname{tr}(H-V)_{-}^{\gamma} \leq L \int_{X} V_{+}^{p} w d \mu
$$

Then one also has

$$
\operatorname{tr}(M-V)_{-}^{\gamma} \leq L\left(\frac{e}{p}\right)^{p} \Gamma(p+1) \int_{X} V_{+}^{p} w d \mu .
$$

We do not know whether the factor $(e / p)^{p} \Gamma(p+1)$ in (4.3) can be omitted. Results from [FrLoWe] about the eigenvalues of the Landau Hamiltonian in a domain (but without potential) seem to indicate that a factor $>1$ is necessary. Our proof of Theorem 4.2 uses some ideas from [Ro] where the case $\gamma=0$ was treated; see also [FrLiSe2] for a result about operators with discrete spectrum.

Remark 4.3. With the same proof one can deduce estimates on $\operatorname{tr} f(M)$ from estimates on $\operatorname{tr} f(H)$ for more general functions $f$. For example, let $d=2$ and $f(t):=|\ln | t||^{-1}$ if $-e^{-1}<t<0, f(t):=1$ if $t \leq-e^{-1}$, and $f(t):=0$ if $t \geq 0$. Then there exists a constant $L$ and for any $q>1$ a constant $L_{q}$ such that for all $l>0$ and $A \in L_{2, \operatorname{loc}}\left(\mathbb{R}^{2}, \mathbb{R}^{2}\right)$,

$$
\begin{aligned}
\operatorname{tr} f\left(l^{2}\left((D-A)^{2}-V\right)\right) \leq L & \int_{|x|<l} V(x)_{+}\left|\log \frac{|x|}{l}\right| d x \\
& +L_{q} \int_{0}^{\infty}\left(\int_{\mathbb{S}} V(r \omega)_{+}^{q} d \omega\right)^{1 / q} r d r .
\end{aligned}
$$

Indeed, this follows by Lemma 4.4 via integration from the $A \equiv 0$ result of [KoVuWe]. 
The key ingredient in the proof of Theorem 4.2 is a bound on the negative eigenvalues of $M-V$ by those of $H-\alpha V$, averaged over all coupling constants $\alpha$. As before, we denote by $N(-\tau, A)$ the number of eigenvalues less than $-\tau$, counting multiplicities, of a self-adjoint operator $A$.

Lemma 4.4. Let $H$ and $M$ be non-negative self-adjoint operators satisfying (4.1) and let $V \geq 0$. Then for any $\tau \geq 0$ and $t>0$ one has

$$
N(-\tau, M-V) \leq t e^{t} \int_{0}^{\infty} N(-\tau, H-\alpha V) e^{-\alpha t} d \alpha .
$$

Proof. Since (4.1) remains valid with $H+\tau$ and $M+\tau$ in place of $H$ and $M$ we need only consider $\tau=0$. Moreover, by an approximation argument we may assume that $V>0$ a.e. We define $h:=V^{-1 / 2} H V^{-1 / 2}$ and $m:=V^{-1 / 2} M V^{-1 / 2}$ via quadratic forms and claim that (4.1) holds with $h$ and $m$ in place of $H$ and $M$. Since this fact is proved in [Ro, Thm. 3] we only sketch the main idea. Indeed, for any $\sigma>0$,

$$
(m+\sigma)^{-1}=V^{1 / 2}(M+\sigma V)^{-1} V^{1 / 2}=\int_{0}^{\infty} V^{1 / 2} \exp (-s(M+\sigma V)) V^{1 / 2} d s,
$$

and by (4.1) and Trotter's product formula $\left|\exp (-s(M+\sigma V)) V^{1 / 2} u\right| \leq \exp (-s(H+$ $\sigma V)) V^{1 / 2}|u|$ a.e. Hence $\left|(m+\sigma)^{-1} u\right| \leq(h+\sigma)^{-1}|u|$ a.e. Iterating this inequality and recalling that $(1+t m / n)^{-n} \rightarrow \exp (-t m)$ strongly as $n \rightarrow \infty$, we obtain (4.1) for $h$ and $m$.

By [Si2, Thm. 2.13] this analog of (4.1) implies that

$$
\operatorname{tr} \exp (-t m)=\|\exp (-t m / 2)\|_{2}^{2} \leq\|\exp (-t h / 2)\|_{2}^{2}=\operatorname{tr} \exp (-t h)
$$

with $\|\cdot\|_{2}$ the Hilbert-Schmidt norm, and hence by the Birman-Schwinger principle,

$$
N(M-V)=N(1, m) \leq e^{t} \operatorname{tr} \exp (-t m) \leq e^{t} \operatorname{tr} \exp (-t h) .
$$

Using the Birman-Schwinger principle once more, we find

$$
\operatorname{tr} \exp (-t h)=t \int_{0}^{\infty} N(\alpha, h) e^{-t \alpha} d \alpha=t \int_{0}^{\infty} N(H-\alpha V) e^{-t \alpha} d \alpha,
$$

proving (4.4).

Proof of Theorem 4.2. By the variational principle we may assume that $V \geq 0$. By Lemma 4.4 one has for any $t>0$,

$$
\begin{aligned}
\operatorname{tr}(M-V)_{-}^{\gamma} & =\gamma \int_{0}^{\infty} N(-\tau, M-V) \tau^{\gamma-1} d \tau \\
& \leq \gamma t e^{t} \int_{0}^{\infty} \int_{0}^{\infty} N(-\tau, H-\alpha V) \tau^{\gamma-1} d \tau e^{-\alpha t} d \alpha \\
& =t e^{t} \int_{0}^{\infty} \operatorname{tr}(H-\alpha V)_{-}^{\gamma} e^{-\alpha t} d \alpha,
\end{aligned}
$$

and by assumption (4.2) the right-hand side can be bounded from above by

$$
L t e^{t}\left(\int_{0}^{\infty} \alpha^{p} e^{-\alpha t} d \alpha\right) \int_{X} V^{p} w d \mu=L t^{-p} e^{t} \Gamma(p+1) \int_{X} V^{p} w d \mu .
$$

Now the assertion follows by choosing $t=p$. 


\section{A Pseudo-Relativistic Model Including Spin}

Throughout this section we assume that $d=3$. The helicity operator $h$ on $L_{2}\left(\mathbb{R}^{3}, \mathbb{C}^{2}\right)$ is defined as the Fourier multiplier corresponding to the matrix-valued function $p \mapsto$ $\sigma \cdot p /|p|$, where $\sigma=\left(\sigma_{1}, \sigma_{2}, \sigma_{3}\right)$ denotes the triple of Pauli matrices. The properties of these matrices imply that $h$ is a unitary and self-adjoint involution. The analog of the Hardy (or Kato) inequality (1.2) is

$$
\int_{\mathbb{R}^{3}}|\xi||\hat{u}(\xi)|^{2} d \xi \geq \tilde{\mathcal{C}} \int_{\mathbb{R}^{3}} \frac{|u(x)|^{2}+|(h u)(x)|^{2}}{2|x|} d x, \quad u \in C_{0}^{\infty}\left(\mathbb{R}^{3}, \mathbb{C}^{2}\right),
$$

with the sharp constant

$$
\tilde{\mathcal{C}}=\frac{2}{2 / \pi+\pi / 2} ;
$$

see $[\mathrm{EvPeSi}]$. Note that this constant is strictly larger than

$$
\mathcal{C}:=\mathcal{C}_{1 / 2,3}=2 / \pi,
$$

which is the constant one would get if $h u$ were replaced by $u$ on the right side of (5.1).

For a function $V$ on $\mathbb{R}^{3}$ taking values in the Hermitian $4 \times 4$ matrices we introduce the non-local potential

$$
\Phi(V):=\frac{1}{2}\left(\begin{array}{c}
1_{L_{2}\left(\mathbb{R}^{3}, \mathbb{C}^{2}\right)} \\
h
\end{array}\right)^{*} V\left(\begin{array}{c}
1_{L_{2}\left(\mathbb{R}^{3}, \mathbb{C}^{2}\right)} \\
h
\end{array}\right),
$$

where $\left(\begin{array}{c}1_{L_{2}\left(\mathbb{R}^{3}, \mathbb{C}^{2}\right)}^{h} \\ h\end{array}\right)$ is considered as an operator from $L_{2}\left(\mathbb{R}^{3}, \mathbb{C}^{2}\right)$ to $L_{2}\left(\mathbb{R}^{3}, \mathbb{C}^{4}\right)$. The operator $\sqrt{-\Delta}-\Phi(V)$ in $L_{2}\left(\mathbb{R}^{3}, \mathbb{C}^{2}\right)$ has been suggested by Brown and Ravenhall as the Hamiltonian of a massless, relativistic spin-1/2 particle in a potential $-V$. It results from projecting onto the positive spectral subspace of the Dirac operator. One of the advantages of this operator over the simpler $\sqrt{-\Delta}-V$ is that it is well-defined for nuclear charges $\alpha Z \leq \tilde{\mathcal{C}}$, which includes all known elements. We refer to [LiSe] for more background about this model. Despite the efforts in [LiSiSo, $\mathrm{BaEv}, \mathrm{HoSi}]$ the problem of stability of matter for the corresponding many-particle system is not yet completely understood and the following result, we believe, might be useful in this respect.

Theorem 5.1. Let $d=3$ and $\gamma>0$. Then there is a constant $\tilde{L}_{\gamma}^{\mathrm{HLT}}$ such that

$$
\operatorname{tr}\left(\sqrt{-\Delta}-\tilde{\mathcal{C}} \Phi\left(|x|^{-1}\right)-\Phi(V)\right)_{-}^{\gamma} \leq \tilde{L}_{\gamma}^{\mathrm{HLT}} \int_{\mathbb{R}^{3}} \operatorname{tr}_{\mathbb{C}^{4}} V(x)_{+}^{\gamma+3} d x .
$$

For the proof of this theorem we need some facts about the partial wave decomposition of the operator $\sqrt{-\Delta}-\tilde{\mathcal{C}} \Phi\left(|x|^{-1}\right)$ from [EvPeSi]. This operator commutes with the total angular momentum operator $\mathbf{J}=\mathbf{L}+\frac{1}{2} \sigma$, where $\mathbf{L}=-i \nabla \times x$, as well as with the operator $\mathbf{L}^{2}$. The subspace corresponding to total angular momentum $j=1 / 2$ is of the form $\mathfrak{H}_{1 / 2,0} \oplus \mathfrak{H}_{1 / 2,1}$, where the subspaces $\mathfrak{H}_{1 / 2, l}$ correspond to the eigenvalues $l(l+1)$ of $\mathbf{L}^{2}$.

The next result, essentially contained in [FrSiWa], says that on the space $\mathfrak{H}_{1 / 2,0} \oplus$ $\mathfrak{H}_{1 / 2,1}$ the operator $\sqrt{-\Delta}-\tilde{\mathcal{C}} \Phi\left(|x|^{-1}\right)$ is controlled by the operator $\sqrt{-\Delta}-\mathcal{C}|x|^{-1}$ with the smaller coupling constant $\mathcal{C}$. (Strictly speaking, the latter operator should be tensored with $1_{\mathbb{C}^{2}}$, but we suppress this if there is no danger of confusion.) 
Lemma 5.2. If $0 \not \equiv \psi \in \mathfrak{H}_{1 / 2,0} \cap C_{0}^{\infty}\left(\mathbb{R}^{3}, \mathbb{C}^{2}\right)$, then

$$
\frac{2}{1+(2 / \pi)^{2}} \geq \frac{\left(\psi,\left(\sqrt{-\Delta}-\tilde{\mathcal{C}} \Phi\left(|x|^{-1}\right)\right) \psi\right)}{\left(\psi,\left(\sqrt{-\Delta}-\mathcal{C}|x|^{-1}\right) \psi\right)} \geq \frac{1}{1+(2 / \pi)^{2}} .
$$

If $0 \not \equiv \psi \in \mathfrak{H}_{1 / 2,1} \cap C_{0}^{\infty}\left(\mathbb{R}^{3}, \mathbb{C}^{2}\right)$, this bound is true provided $\left(\psi,\left(\sqrt{-\Delta}-\mathcal{C}|x|^{-1}\right) \psi\right)$ is replaced by $\left(h \psi,\left(\sqrt{-\Delta}-\mathcal{C}|x|^{-1}\right) h \psi\right)$.

Proof of Lemma 5.2. We prove the assertion only for $l=1$ since the lower bound for $l=0$ is contained in [FrSiWa, Lemma 2.7] and the upper bound is proved as below. By orthogonality we may assume that the Fourier transform of $\psi$ is of the form $\hat{\psi}(\xi)=|\xi|^{-2} g(|\xi|) \Omega_{1 / 2,1, m}\left(\frac{\xi}{|\xi|}\right)$, where $m \in\{-1 / 2,1 / 2\}$ and $\Omega_{1 / 2,1, m}$ are explicit functions in $L_{2}\left(\mathbb{S}^{2}, \mathbb{C}^{2}\right)$. By the properties of these functions one has $\widehat{h \psi}(\xi)=$ $-|\xi|^{-2} g(|\xi|) \Omega_{1 / 2,0, m}\left(\frac{\xi}{|\xi|}\right)$. The ground state representation from [FrSiWa, Lemma 2.6] reads

$$
\begin{aligned}
\left(\psi,\left(\sqrt{-\Delta}-\tilde{\mathcal{C}} \Phi\left(|x|^{-1}\right)\right) \psi\right) & =\frac{\tilde{\mathcal{C}}}{2 \pi} \int_{0}^{\infty} \int_{0}^{\infty}|g(p)-g(q)|^{2} \tilde{k}\left(\frac{1}{2}\left(\frac{p}{q}+\frac{q}{p}\right)\right) \frac{d p}{p} \frac{d q}{q} \\
\left(h \psi,\left(\sqrt{-\Delta}-\mathcal{C}|x|^{-1}\right) h \psi\right) & =\frac{\mathcal{C}}{2 \pi} \int_{0}^{\infty} \int_{0}^{\infty}|g(p)-g(q)|^{2} k\left(\frac{1}{2}\left(\frac{p}{q}+\frac{q}{p}\right)\right) \frac{d p}{p} \frac{d q}{q},
\end{aligned}
$$

where $\tilde{k}(t)=\frac{1}{2}\left(Q_{0}(t)+Q_{1}(t)\right), k(t)=Q_{0}(t)$, and $Q_{l}$ are the Legendre functions of the second kind [AbSt, 8.4]. The assertion now follows from the fact that $Q_{0} \geq Q_{1} \geq 0$.

Proof of Theorem 5.1. We first claim that for any $0<t<1 / 2$ there is a $\tilde{K}_{t}>0$ such that

$$
\sqrt{-\Delta}-\tilde{\mathcal{C}} \Phi\left(|x|^{-1}\right) \geq \tilde{K}_{t} l^{-1+2 t}(-\Delta)^{t}-l^{-1}, \quad l>0 .
$$

Indeed, it follows from Lemma 5.2 and (1.8) that on $\mathfrak{H}_{1 / 2,0} \oplus \mathfrak{H}_{1 / 2,1}$ one has for any $0<t<1 / 2$,

$$
\sqrt{-\Delta}-\tilde{\mathcal{C}} \Phi\left(|x|^{-1}\right) \geq\left(1+(2 / \pi)^{2}\right)^{-1}\left(K_{t} l^{-1+2 t}(-\Delta)^{t}-l^{-1}\right), \quad l>0 .
$$

On the other hand, the arguments of [EvPeSi] show that there exists a constant $\tilde{\mathcal{C}}^{\prime}>\tilde{\mathcal{C}}$ such that $\sqrt{-\Delta} \geq \tilde{\mathcal{C}}^{\prime} \Phi\left(|x|^{-1}\right)$ on $\left(\mathfrak{H}_{1 / 2,0} \oplus \mathfrak{H}_{1 / 2,1}\right)^{\perp}$. Hence on that space

$$
\begin{aligned}
\sqrt{-\Delta}-\tilde{\mathcal{C}} \Phi\left(|x|^{-1}\right) & \geq \frac{\tilde{\mathcal{C}}^{\prime}-\tilde{\mathcal{C}}}{\tilde{\mathcal{C}}^{\prime}} \sqrt{-\Delta} \\
& \geq \frac{\tilde{\mathcal{C}}^{\prime}-\tilde{\mathcal{C}}}{\tilde{\mathcal{C}}^{\prime}}\left(\frac{1}{2 t} l^{-1+2 t}(-\Delta)^{t}-\frac{1-2 t}{2 t} l^{-1}\right), \quad l>0 .
\end{aligned}
$$

This proves (5.3).

Given (5.3), the proof of (5.2) is similar to that of (1.5). We may assume that $V(x)=$ $v(x) I_{\mathbb{C}^{4}}$ for a non-negative, scalar function $v$ (otherwise, replace $V(x)$ by $v(x) I_{\mathbb{C}^{4}}$, where $v(x)$ is the operator norm of the $4 \times 4$ matrix $\left.V(x)_{+}\right)$. For a given $l>0$ and 
$0<t<1 / 2$ we introduce the operator $H:=\tilde{K}_{t} l^{-1+2 t}(-\Delta)^{t}-v-l^{-1}$ in $L_{2}\left(\mathbb{R}^{3}, \mathbb{C}\right)$. Then according to (5.3) one has

$$
\begin{aligned}
N\left(-\tau, \sqrt{-\Delta}-\tilde{\mathcal{C}} \Phi\left(|x|^{-1}\right)-\Phi(V)\right) & \leq N\left(-\tau, \frac{1}{2}\left(H \otimes 1_{\mathbb{C}^{2}}+h\left(H \otimes 1_{\mathbb{C}^{2}}\right) h\right)\right) \\
& \leq 4 N(-\tau, H) .
\end{aligned}
$$

In the last inequality we used that $N\left(-\tau, \frac{1}{2}(A+B)\right) \leq N(-\tau, A)+N(-\tau, B)$ for any self-adjoint, lower semi-bounded operators $A$ and $B$, which follows from the variational principle. Now one can proceed in the same way as in the proof of (1.5).

Acknowledgement. The author would like to thank E. Lieb and R. Seiringer for very fruitful discussions, as well as J. P. Solovej, T. Østergaard Sørensen and W. Spitzer for useful correspondence. Support through DAAD grant D/06/49117 and U.S. National Science Foundation grant PHY 0652854 is gratefully acknowledged.

\section{References}

[AbSt] Abramowitz, M., Stegun, I.A.: Handbook of mathematical functions with formulas, graphs, and mathematical tables. Reprint of the 1972 edition. New York: Dover Publications, 1992

[AvHeSi] Avron, J., Herbst, I., Simon, B.: Schrödinger operators with magnetic fields. I. general interactions. Duke Math. J. 45(4), 847-883 (1978)

[BaEv] Balinsky, A.A., Evans, W.D.: Stability of one-electron molecules in the Brown-Ravenhall model. Commun. Math. Phys. 202(2), 481-500 (1999)

[Cw] Cwikel, M.: Weak type estimates for singular values and the number of bound states of schrödinger operators. Ann. Math. 106, 93-102 (1977)

[Da] Daubechies, I.: An uncertainty principle fermions with a generalized kinetic energy. Commun. Math. Phys. 90, 511-520 (1983)

[Do] Donoghue, W.F.: Monotone matrix functions and analytic continuation. New York-Heidelberg, Springer, 1974

[EkFr] Ekholm, T., Frank, R.L.: On Lieb-Thirring inequalities for Schrödinger operators with virtual level. Commun. Math. Phys. 264(3), 725-740 (2006)

[EvPeSi] Evans, W.D., Perry, P., Siedentop, H.: The spectrum of relativistic one-electron atoms according to Bethe and Salpeter. Commun. Math. Phys. 178(3), 733-746 (1996)

[FrLiSe1] Frank, R.L., Lieb, E.H., Seiringer, R.: Hardy-Lieb-Thirring inequalities for fractional Schrödinger operators. J. Amer. Math. Soc. 21(4), 925-950 (2008)

[FrLiSe2] Frank, R.L., Lieb, E.H., Seiringer, R.: Stability of relativistic matter with magnetic fields for nuclear charges up to the critical value. Commun. Math. Phys. 275(2), 479-489 (2007)

[FrLoWe] Frank, R.L., Loss, M., Weidl, T.: Pólya's conjecture in the presence of a constant magnetic field. J. Eur. Math. Soc., to appear

[FrSiWa] Frank, R.L., Siedentop, H., Warzel, S.: The energy of heavy atoms according to Brown and Ravenhall: The Scott correction. http://arxiv.org/abs/0805.4441v2[math-ph], 2008

[He] Herbst, I.W.: Spectral theory of the operator $\left(p^{2}+m^{2}\right)^{1 / 2}-z e^{2} / r$. Commun. Math. Phys. 53(3), 285-294 (1977)

[HoSi] Hoever, G., Siedentop, H.: Stability of the Brown-Ravenhall operator. Math. Phys. Electron. J. 5 ,11 pp (1999)

[Hu] Hundertmark, D.: Some bound state problems in quantum mechanics. In: Spectral theory and mathematical physics: a Festschrift in honor of Barry Simon's 60th birthday, Proc. Sympos. Pure Math. 76, Part 1, Providence, RI: Amer. Math. Soc., 2007, pp. 463-496

[KoPeSe] Kovalenko, V.F., Perel'muter, M.A., Semenov, Ya.A.: Schrödinger operators with $l_{w}^{l / 2}\left(\mathbb{R}^{l}\right)-$ potentials. J. Math. Phys. 22(5), 1033-1044 (1981)

[KoVuWe] Kovařík, H., Vugalter, S., Weidl, T.: Spectral estimates for two-dimensional Schrödinger operators with application to quantum layers. Commun. Math. Phys. 275(3), 827-838 (2007)

[LaWe] Laptev, A., Weidl, T.: Recent results on Lieb-Thirring inequalities. Journées 'Équations aux Dérivées Partielles' (La Chapelle sur Erdre, 2000), Exp. No. XX, Nantes: Univ. Nantes, 2000

[Li] Lieb, E.H.: Flux phase of the half-filled band. Phys. Rev. Lett. 73, 2158-2161 (1994)

[LiLo] Lieb, E.H., Loss, M.: Analysis. Second edition. Graduate Studies in Mathematics 14, Providence, RI: Amer. Math. Soc., 2001

[LiSe] Lieb, E.H., Seiringer, R.: The stability of matter. In preparation 
[LiSiSo] Lieb, E.H., Siedentop, H., Solovej, J.P.: Stability and instability of relativistic electrons in classical electromagnetic fields. J. Stat. Phys. 89(1-2), 37-59 (1997)

[LiTh] Lieb, E.H., Thirring, W.: Inequalities for the moments of the eigenvalues of the Schrödinger Hamiltonian and their relation to Sobolev inequalities. Studies in Mathematical Physics, Princeton, NJ: Princeton University Press, 1976, pp. 269-303

[LiYa] Lieb, E.H., Yau, H.-T.: The stability and instability of relativistic matter. Commun. Math. Phys. 118(2), 177-213 (1988)

[Ro] Rozenblum, G.: Domination of semigroups and estimates for eigenvalues. St. Petersburg Math. J. 12(5), 831-845 (2001)

[Si1] Simon, B.: Maximal and minimal Schrödinger forms. J. Oper. Th. 1(1), 37-47 (1979)

[Si2] Simon, B.: Trace ideals and their applications. Second edition, Mathematical Surveys and Monographs 120, Providence, RI: Amer. Math. Soc., 2005

[SoSøSp] Solovej, J.P., Sørensen, Ø.T., Spitzer, W.: The relativistic Scott correction for atoms and molecules. http://arxiv.org/abs/0808.2163v1[math-ph], 2008

Communicated by B. Simon 